\title{
PERSPECTIVE
}

\section{Decanalization, brain development and risk of schizophrenia}

\author{
JJ McGrath ${ }^{1,2,3}$, AJ Hannan ${ }^{4}$ and G Gibson ${ }^{5}$
}

Waddington's original description of canalization refers to the ability of an organism to maintain phenotypic fidelity in the face of environmental and/or genetic perturbation. Development of the human brain requires exposure to a 'wild-type' environmentone that supports the optimal set of instructions for development. Recently derived brain structures in our species, such as the expanded neocortex, may be more vulnerable to decanalization because there has been insufficient time to evolve buffering capacity. On the basis of modern notions of decanalization, we provide perspectives on selected environmental and genetic risk factors for schizophrenia, and we discuss strengths and weaknesses of this conceptual framework. We argue that if we are to build a solid foundation for translational psychiatry, we must explore models that attempt to capture the complexity of the interaction between genetic and non-genetic risk factors in mediating and modulating brain development.

Translational Psychiatry (2011) 1, e14; doi:10.1038/tp.2011.16; published online 28 June 2011

\section{Introduction}

Conrad Waddington provided a powerful metaphor to help conceptualize the process of development. He described ontogeny as a contoured and sloping surface, underpinned by complex interactions between genetic and non-genetic factors during development. The topography of this landscape actively channels (that is, canalizes) the developmental trajectory of an organism toward an optimal phenotype. ${ }^{1}$ In this imaginary landscape, the depth of a channel reflects the buffering capacity available to an organism-deep channels are more likely to result in the desired phenotype in the face of genetic or non-genetic perturbation. Implicit in the model is the assumption that evolution sculpts the landscape in response to exposures most regularly encountered in the environment. When an organism moves out of this adaptive niche, the capacity to buffer the developmental trajectory can be compromised. Waddington described this process as decanalization. ${ }^{1}$

\section{Modern perspectives of decanalization}

In recent years, evolutionary geneticists have reconceptualized canalization to emphasize the distinction between (a) developmental or physiological robustness (which occurs across ontogeny) and (b) the evolution of robustness (which occurs across phylogeny). The result is a conceptual framework that places canalization in the context of genotype-byenvironment $(G \times E)$ and/or genotype-by-genotype (epistatic) interactions. Under long-term stabilizing selection, genetic systems are expected to evolve to a state of increased stability, such that the proportion of less 'fit' individuals is minimized. Consequently, canalization is now seen as a population-level phenomenon, and decanalization is said to occur when the variability of a trait at the population level increases after some perturbation. ${ }^{2}$ When a population is moved out of the adaptive niche, the variability of certain traits may increase. As a consequence of this decanalization, a greater proportion of the population may cross a phenotypic threshold (that is, more disease outcomes). In this scenario, the mean value for a quantitative trait may remain unchanged, while the variance increases. This is displayed in Figure 1. Apart from an increase in variance, $G \times E$ interactions may also influence the incidence of a disease by shifting the entire distribution in either direction.

The classical example of decanalization is the Tabby mutant mouse. ${ }^{3}$ Normal mice almost always have 17 or 18 vibrissae on either side of the snout, but Tabby mice have anywhere from 5 to 20 such whiskers. In this case, the variability has increased, leading to the inference that there is a general loss of developmental stability. The mutation causes a loss of canalization. Another example is the production of photoreceptors in flies, where under normal circumstances, each of the several hundred ommatidia of a compound eye has exactly 8 photoreceptor cells, but in the presence of a gain-of-function allele of the epidermal growth factor receptor, the number varies from 8 to 12 or more depending on the genetic background. ${ }^{4}$

It has been suggested that canalization is an inherent feature of highly redundant gene networks. ${ }^{5}$ In addition,

${ }^{1}$ Queensland Brain Institute, University of Queensland, St Lucia, QLD, Australia; ${ }^{2}$ Queensland Centre for Mental Health Research, The Park Centre for Mental Health, Wacol, QLD, Australia; ${ }^{3}$ Department of Psychiatry, University of Queensland, St Lucia, QLD, Australia; ${ }^{4}$ Howard Florey Institute, Florey Neuroscience Institutes, University of Melbourne, Melbourne, VIC, Australia and ${ }^{5}$ School of Biology, Georgia Institute of Technology, Atlanta, GA, USA

Correspondence: Professor J McGrath, Queensland Brain Institute, University of Queensland, Brisbane, St Lucia, QLD 4072, Australia.

E-mail: john_mcgrath@qcmhr.uq.edu.au

Keywords: environmental risk factors; neurodevelopment; schizophrenia

Received 23 May 2011; accepted 01 June 2011 


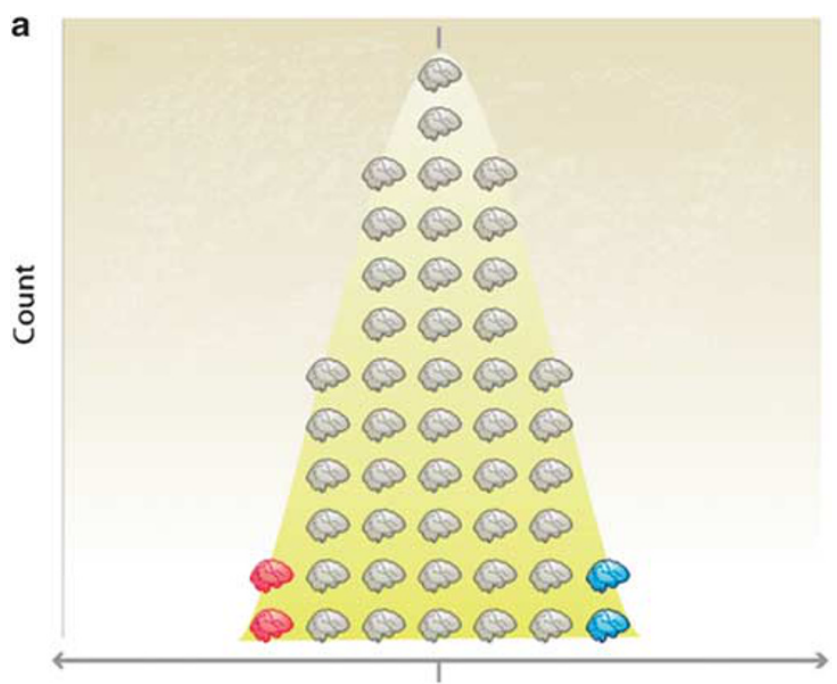

Brain phenotype

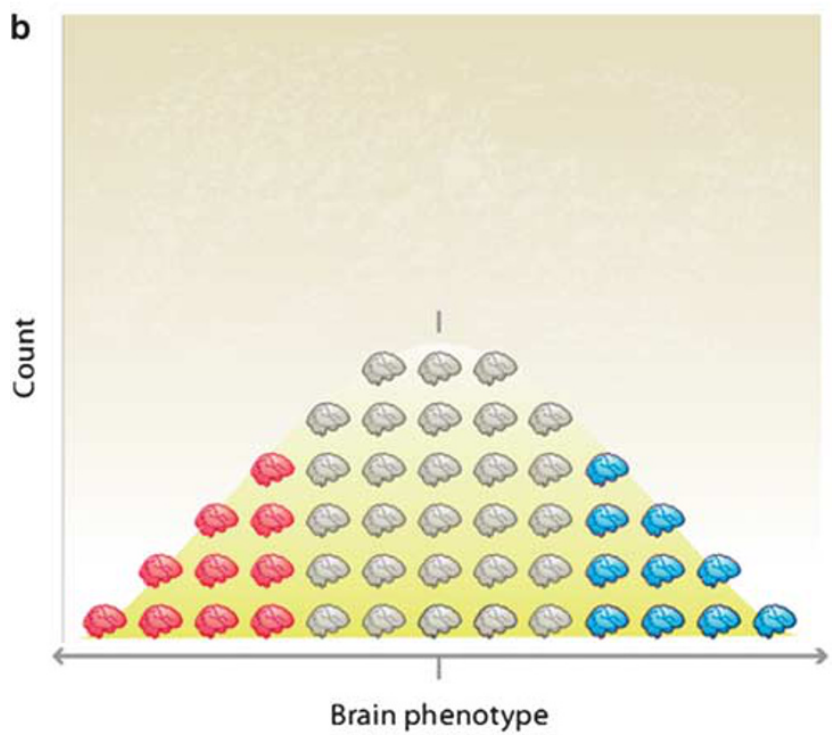

Figure 1 The distribution of quantitative brain phenotypes. The upper panel (a) shows a population with a small proportion of deviant outcomes (shown in red and blue). The lower panel (b) shows how decanalization of a brain phenotype can leave the mean value unaffected but result in a greater proportion of deviant outcomes (shown in red and blue). Note that the increase in variance need not be symmetric for decanalization to occur.

neutral genetic variation may add to the capacity of an organism to buffer events not frequently encountered in the 'wild-type' environment. Although these polymorphisms are usually phenotypically silent (that is, cryptic), they may act as a buffer to allow an organism exposed to an unexpected environmental stress, or to a de novo mutation, to resist the transition to alternative phenotypes. ${ }^{6}$

\section{Is the brain highly vulnerable to decanalization?}

Many factors influence brain development, including the timing and location of cell birth (which influences cell fate), ${ }^{7}$ and positional clues, such as contact with adjacent cells and gradients of various trophic agents, which influence cell and axonal migration. Although many of these features are generic across different organs, the development of the brain is particularly sensitive to instructions from the internal and external environments.

Both innate and activity-dependent neuronal activity have a critical role in optimal brain development. ${ }^{8}$ With respect to activity-dependent processes, the brain extracts information from the environment and uses this information to dynamically shape aspects of cellular and subcellular structure. Landmark experiments performed over 30 years ago demonstrated that the fate map for brain development has critical windows, during which sensory input is necessary for optimal development. ${ }^{9}$ Key elements of brain development have evolved to be experience expectant-the environment needs to provide the instructions for optimal brain development. $^{10}$

The importance of optimal environmental input for brain function has been demonstrated with rodent models of psychiatric and neurological disorders. ${ }^{11,12}$ Standard animal housing used by the vast majority of biomedical researchers lacks sensorimotor stimulation compared to 'wild-type' environments (for example, an environment where these rodents originally evolved). It has also been noted that laboratory animals in the so-called 'enriched environment' housing conditions do better on a range of outcomes compared with 'standard' housing conditions. Strikingly, the neurodegenerative phenotype (including cognitive, affective and motor deficits) of Huntington's disease transgenic mice is delayed when they are housed in an enriched environment. ${ }^{13-15}$ This suggests that the impact of the mutation has been ameliorated by the enriched environment setting, or conversely that sensorimotor deprivation within 'standard housing' conditions may have accelerated the disease process. Recent evidence suggests that an enriched environment can ameliorate the behavioral phenotype associated with a phospholipase C- $\beta 1$ knockout mouse, which has been proposed as an informative animal model for schizophrenia research. ${ }^{16}$ Although simple phenotypes are unaffected by standard laboratory housing (for example, coat color), we argue that exposure to these conditions during development can decanalize experience-dependent behavioral phenotypes. Just as transgenic animals have altered instructional vectors as a result of perturbed input from particular genes, 'knocking out' adequate sensory and cognitive stimulation results in an animal with an environmentally edited developmental trajectory.

We postulate that the brain is particularly vulnerable to decanalization based on the following features: (a) the brain is a highly complex organ requiring instructional input from expression of over half of the genome, ${ }^{17}$ (b) compared with closely related species, the hominid neocortex has expanded considerably in our species; hence, there may have been insufficient time to evolve robust cortical developmental trajectories, (c) the brain is intensely reactive to the environment and is experience expectant, and disruption of this input can impact on behavioral phenotypes and (d) the brain has tightly-regulated critical windows of development; thus, there are few opportunities to compensate for perturbation. 
a

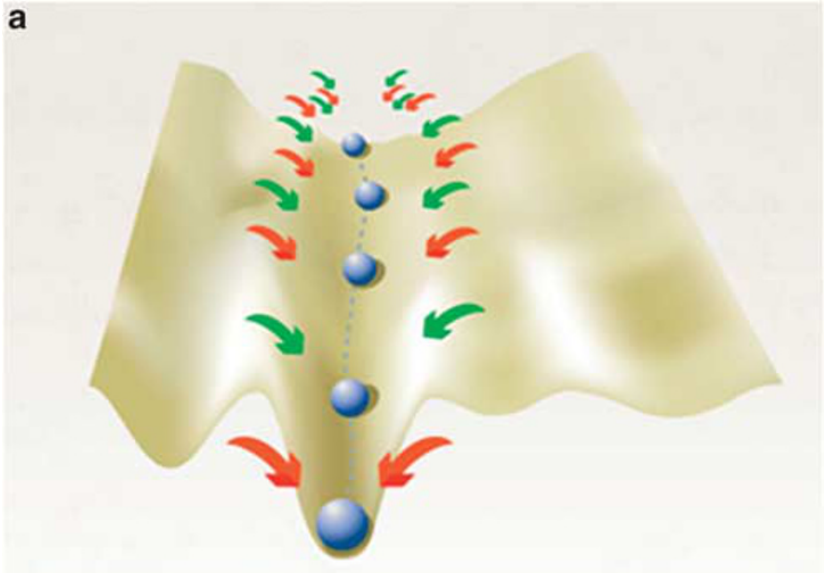

b

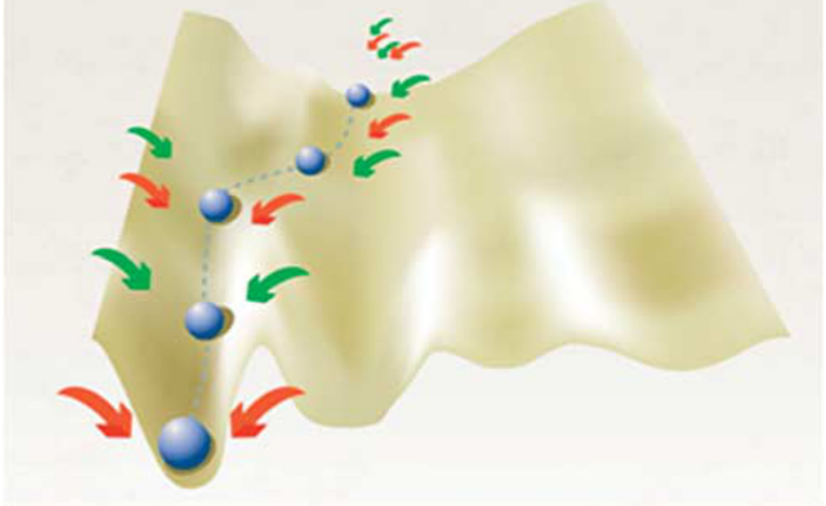

C

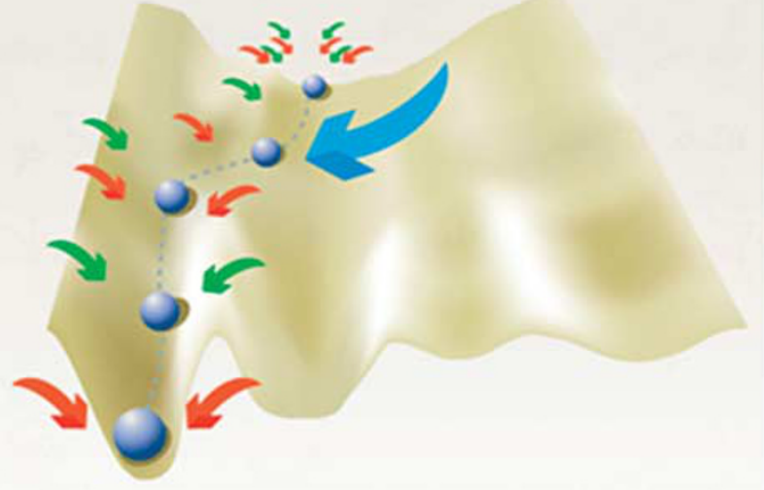

Figure 2 The epigenetic landscape. The upper panel (a) shows an optimal developmental trajectory, with a range of expected instructions (red and green arrows) contributing over time to maintain the desired trajectory. The middle panel (b) shows how the absence of expected instructions during early development (unbalanced arrows in the top of the figure) can decanalize development. The lower panel (c) shows how an unexpected instruction (large blue arrow) can also lead to decanalization of development.

Figure 2 shows an updated interpretation of the Waddington's epigenetic landscape, with the smaller arrows representing expected instructional vectors guiding brain development. Figure 2a displays an optimal trajectory with a compendium of expected genetic and environmental instructions. Suboptimal developmental trajectories can result from the absence of expected (Figure $2 b$ ) or the presence of unexpected (Figure $2 \mathrm{c}$ ) instructional vectors.

\section{The causes of schizophrenia-clues from the environment}

Schizophrenia is a poorly understood group of brain disorders that impacts on perception, cognition and emotion. Although the most prominent symptoms usually emerge in the second and third decade of life, there is convergent evidence indicating that schizophrenia is associated with early disruption of brain development. ${ }^{18}$

Some of the most robust risk factors for schizophrenia are related to non-genetic exposures. ${ }^{19,20}$ A startling finding to emerge from recent schizophrenia epidemiology relates to the very high rates in some migrant groups. ${ }^{21,22}$ A UK-based multi-site incidence study ${ }^{23}$ reported a ninefold increased risk of schizophrenia in African Caribbeans and nearly a sixfold increased risk in black migrants from Africa. The risk tends to be higher in second-generation migrants (that is, those born in the host nation) compared with first-generation migrants (who can arrive in the host nation as children or adults). Incidence studies in the countries of origin of these migrants do not indicate particularly high rates of schizophrenia in these ethnic groups, ${ }^{24}$ and the increased risk cannot be explained by systematic under- or overreporting in the country of origin or host nation. The data strongly suggest that the increased risk in the migrant groups is due to environmentally mediated exposures. Individuals with dark skin are particularly at risk. ${ }^{22}$ As minority ethnic groups can be exposed to chronic stress or 'social defeat', the role of social biology has influenced recent schizophrenia research. ${ }^{20,25}$

Another pointer to environmental factors contributing to risk of schizophrenia has come from studies linking place of birth and risk of schizophrenia. For example, population-based studies from Holland ${ }^{26}$ and Denmark ${ }^{27}$ have found a twofold increased risk of developing schizophrenia in those born and raised in the city compared with rural settings. The mechanism underpinning this association remains poorly understood. As many populations are already strongly urbanized, a large proportion of the population is exposed to this yet-to-beidentified factor.

It has been proposed that developmental vitamin D deficiency may contribute to the increased risk of schizophrenia in dark-skinned migrants and those living in crowded urban settings. ${ }^{28}$ Both dark skin and urban residence are associated with an increased risk of hypovitaminosis D. ${ }^{29}$ Developmental vitamin $D$ deficiency may also underlie the well-replicated season of birth effect found in schizophrenia (those born in winter and spring have a small but significant increased risk of schizophrenia). ${ }^{30}$ Vitamin D is a potent prodifferentiation agent and can influence brain functioning via many different pathways. ${ }^{31}$ The vitamin $D$ receptor and the enzyme required for the manufacture of the hormonally active form of vitamin $D$ have been identified in the human brain, ${ }^{32}$ and there is evidence from rodent models demonstrating that transient prenatal vitamin $\mathrm{D}$ deficiency results in persistent changes in adult brain structure, neurochemistry and behavior. $^{33}$ Our species has evolved in settings where vitamin $\mathrm{D}$ is abundant, and migration to colder climates and 
urban-related lifestyle factors have left modern humans at increased risk of hypovitaminosis $D$.

Inspired by the conceptual framework described above, we propose that the absence of adequate vitamin $D$ levels during development deprives the organism of expected instructions from the environment. We speculate that (a) developmental vitamin $D$ deficiency may underlie key features of the epidemiology of schizophrenia, (b) the vitamin D deficiency during development decanalizes brain development and (c) schizophrenia is a behavioral phenotype associated with increased variance associated with this decanalization of brain development. We speculate that prenatal vitamin D deficiency would be one of many different exposures that could decanalize brain development and lead to an increased susceptibility to schizophrenia. van Os et al. ${ }^{20}$ have argued that genetic factors may render some individuals differentially sensitivity to environments of victimization and social exclusion, which in turn leads to an increased risk of schizophrenia. In these scenarios, stress-related mechanisms may decanalize optimal brain function, particularly during critical periods of brain maturation during adolescence. ${ }^{34}$ The adolescent brain may also be sensitive to the impact of cannabis, which has been associated with an increased risk of schizophrenia. ${ }^{35,36}$

\section{The causes of schizophrenia-clues from genetics}

The genetic contribution to different diseases will vary according to the degree of canalization, which in turn will be a function of the genetic architecture of the disease, the history and intensity of stabilizing selection, and the level of environmental perturbation. ${ }^{2}$ Given the high heritability of schizophrenia, ${ }^{37}$ it was expected that genome-wide association studies would reveal dozens of common susceptibility alleles. However, examination of over 15000 cases has revealed only the major histocompatibility complex as a replicated susceptibility locus, ${ }^{38-40}$ and the surprising conclusion is that common alleles of even moderate effect collectively account for no more than a few percent of case-control status in a sample of $>3000$ cases and controls. ${ }^{39}$ To account for this 'missing heritability', opinion has shifted toward two alternate explanations. The International Schizophrenia Consortium has proposed that there are in fact thousands of variants including common variants of very small effect that account for at least a third of susceptibility. ${ }^{39}$ The false-positive rate is so high for small-effect alleles that it is impossible to assign risk to any particular variants, but it can be inferred from the collective distribution of scores that they exist. ${ }^{41}$ This so-called 'polygenic model' encompasses a genetic architecture with contributions of both rare and common variants, consistent with the finding of a handful of rare copy number variants that are clearly associated with schizophrenia. ${ }^{42}$ Other researchers interpret the copy number variant results to support their hypothesis that only rare variants can explain schizophrenia. ${ }^{43}$ This conclusion is perhaps buttressed by exon resequencing studies of $\mathrm{X}$-linked mental retardation that establish a major role for rare mutations in that neurological condition. ${ }^{44}$

Modern perspectives of decanalization provide various clues that can help guide future research. The first is that current case-control approaches to detect association with disease may be more likely to succeed in particular high-risk groups. If cryptic variation is exposed by environmental exposures, such as vitamin D deficiency, stress or cannabis use, then allelic effects should be larger in populations where these exposures are prevalent. On the other hand, if the influence of the allele is variable across the population as a whole in the presence of hidden environmental or population structure, then its total contribution may be diminished below genome-wide significance thresholds. In general, genomewide association study approaches are underpowered to detect all but the largest genotype-by-environment interactions; hence, direct genotypic detection of canalizing alleles is not expected to be straightforward.

Second, it is important to recognize that there is no a priori reason why rare variants of large effect should have consistent effects across families and/or populations. It is intuitive to suppose that large-effect mutations will have more consistent penetrance and expressivity than weak effect polymorphisms, but model organism research has established that even largeeffect variants can be strongly modified by the genetic background and environment. ${ }^{6}$ Even the master regulator of eye development in Drosophila, eyeless, can be suppressed by growth of the flies at different humidity. ${ }^{45}$

Third, this model makes no predictions about whether risk factors will be derived or ancestral. Again, it is intuitive to suppose that a debilitating condition such as schizophrenia could be attributed, in part, to recently derived rare mutations. However, common variants of small effect that make a contribution may be ancestral or derived. Exposure to novel environments may result in ancestral variants being linked to disease states. It is well known that many of the type 2 diabetes risk alleles are ancestral, and that derived protective alleles have risen to high frequencies in some populations. ${ }^{2}$ It is thus important to understand how genetic variation impacts brain development, in general, and, in particular, the robustness of these trajectories.

\section{Decanalization as a conceptual framework for translational psychiatry}

Theories related to decanalization have been previously used to conceptualize the development of psychopathology. ${ }^{46}$ In particular, Woolf discussed the neurodevelopmental hypothesis of schizophrenia from the perspective of decanalization. ${ }^{47}$ With respect to non-genetic risk factors, conceptual frameworks based on decanalization remind us how brain development can be altered by environmental editing. The absence of key environmental instructions can disrupt brain development, just as an unexpected environmental exposures or de novo mutation may also be sufficient to decanalize brain development. The resultant phenotypes reflect the buffering capacity of the epigenetic landscape to fully or partially 'rescue' the developmental trajectory. Currently, there is considerable interest in how epigenetic factors (for example, changes in methylation or histone modification) can influence the onset of neurodevelopmental and neuropsychiatric disorders. ${ }^{48}$ Early environmental exposures such as postnatal maternal care ${ }^{49}$ can permanently influence epigenetic status of key genes involved in stress response. Prenatal diet can also disrupt epigenetic processes in a 
permanent manner that alter key phenotypes. ${ }^{50,51}$ Understanding these processes may translate to interventions that protect early brain development from adverse exposure (that is, primary prevention).$^{52}$

Waddington's metaphor can also help remind the research community about less readily observable features of brain development. First, the epigenetic landscape is not static or fixed. The contours are reactive-they are influenced by instructional vectors that are continuously being refreshed. Second, the model foregrounds the shared and iterative nature of heritable and environmental instructions in real-time elaboration of the developmental trajectory. For the researcher interested in understanding the causes of neurodevelopmental disorders such as schizophrenia, it shifts the focus away from single causes to broader domains of interest. For example, the expression of classes of genes that contribute to the canalization of cortical development would be an appropriate category of observation (rather than one particular gene). Similarly, the absence of expected environmental instructions warrants equal scrutiny alongside the presence of unexpected environmental exposures. The model may also help identify clusters of neuropsychiatric conditions that emerge from shared decanalization events. Clinical disorders with widely varying surface-level behavioral phenotypes (for example, schizophrenia and autism) may share early decanalization events. The separate developmental trajectories leading to divergent phenotypes may be the result of chance events or different heritable vectors that influence the individual epigenetic landscape. Unraveling the upstream factors influencing adult behavioral phenotypes will be a major challenge.

\section{Limitations of the conceptual framework}

Loose conceptual frameworks, however, inspiring on first inspection, do not always translate to proximal and readily testable hypotheses. Similar to the field of evolutionary developmental biology in general, these notions provide a framework upon which to contextualize empirical findings and help generate future specific hypotheses. It can be argued that these notions add little to existing neuroscience concepts (for example, brain plasticity in response to the environment and critical periods of brain development). Although the framework may force the researchers to consider causality from wider categories of observation, this does not necessarily reduce the search space from which to select the candidate genes or exposures. Despite the initial appeal of the framework, it does not offer specific detail into the underlying calculus that integrates developmental pathways (in contrast to quantitative genetics that has a strong statistical framework).

Furthermore, brain-related phenotypes, in general, and neuropsychiatric phenotypes, in particular, are emergent properties of staggering biocomplexity. Simple Waddingtoninspired models will not be sufficient to unravel this complexity. We must have a sober appreciation of how difficult it will be to reverse engineer deeply canalized systems. When developmental systems fail, canalization allows the organism to call up other routines to maintain output. If these systems cannot cope, features of the system may undergo 'graceful degradation'. This term (from computer programming) is used to describe the ability of software to continue operating with reduced function rather than 'crash'. Perhaps the symptoms of schizophrenia are best conceptualized as the graceful degradation of failing, complex cognitive systems.

\section{Conclusions}

If we are to bridge the 'valley of death' between basic neuroscience and translational psychiatry, ${ }^{53}$ we must first understand how genetic and non-genetic risk factors influence brain development. We propose that recent developments in Waddington's concept of decanalization may have heuristic value for psychiatric research. With respect to the epidemiology of schizophrenia, we outline how one candidate environmental risk factor, low prenatal vitamin $D$, can result in environmental editing of brain development. As a result, the decanalized brain is prone to greater variance, resulting in suboptimal phenotypes such as schizophrenia. With respect to the genetics of schizophrenia, understanding how decanalization can unmask cryptic genetic variation provides a conceptual framework that can inform our understanding of the genetic architecture of schizophrenia. We hope that these models can inspire the research community to generate new testable hypotheses to elucidate the causes of poorly understood brain disorders such as schizophrenia.

\section{Conflict of interest}

The authors declare no conflict of interest.

Acknowledgements. We are grateful to Naomi Wray and Linda Richards, who provided helpful comments on early drafts of the manuscript. JM has been supported by the National Health and Medical Research Council and a Rockefeller Bellagio Residency. AJH is supported by the National Health and Medical Research Council and an Australian Research Council Future Fellowship. GG was supported by an Australian Professorial Fellowship.

1. Waddington $\mathrm{CH}$. Canalization of development and genetic assimilation of acquired characters. Nature 1959; 183: 1654-1655.

2. Gibson G. Decanalization and the origin of complex disease. Nat Rev Genet 2009; 10: 134-140.

3. Dun RB, Fraser AS. Selection for an invariant character; vibrissa number in the house mouse. Nature 1958; 181: 1018-1019.

4. Dworkin I, Palsson A, Birdsall K, Gibson G. Evidence that Egfr contributes to cryptic genetic variation for photoreceptor determination in natural populations of Drosophila melanogaster. Curr Biol 2003; 13: 1888-1893.

5. Bergman A, Siegal ML. Evolutionary capacitance as a general feature of complex gene networks. Nature 2003; 424: 549-552.

6. Gibson G, Dworkin I. Uncovering cryptic genetic variation. Nat Rev Genet 2004; 5: 681-690

7. McConnell SK. The determination of neuronal fate in the cerebral cortex. TINS 1989; 12 : 342-349

8. Rakic P. Evolution of the neocortex: a perspective from developmental biology. Nat Rev Neurosci 2009; 10: 724-735.

9. Hubel DH, Wiesel TN. The period of susceptibility to the physiological effects of unilateral eye closure in kittens. J Physiol 1970; 206: 419-436.

10. Greenough WT, Black JE, Wallace CS. Experience and brain development. Child Dev 1987; 58: 539-559.

11. Sale A, Berardi N, Maffei L. Enrich the environment to empower the brain. Trends Neurosci 2009; 32: 233-239.

12. Nithianantharajah J, Hannan AJ. Enriched environments, experience-dependent plasticity and disorders of the nervous system. Nat Rev Neurosci 2006; 7: 697-709.

13. van Dellen A, Grote HE, Hannan AJ. Gene-environment interactions, neuronal dysfunction and pathological plasticity in Huntington's disease. Clin Exp Pharmacol Physiol 2005; 32: 1007-1019. 
14. Hockly E, Cordery PM, Woodman B, Mahal A, van Dellen A, Blakemore C et al. Environmental enrichment slows disease progression in R6/2 Huntington's disease mice. Ann Neurol 2002; 51: 235-242.

15. Nithianantharajah J, Barkus C, Murphy M, Hannan AJ. Gene-environment interactions modulating cognitive function and molecular correlates of synaptic plasticity in Huntington's disease transgenic mice. Neurobiol Dis 2008; 29: 490-504.

16. McOmish CE, Burrows E, Howard M, Scarr E, Kim D, Shin HS et al. Phospholipase C-beta1 knockout mice exhibit endophenotypes modeling schizophrenia which are rescued by environmental enrichment and clozapine administration. Mol Psychiatry 2008; 13: $661-672$.

17. Colantuoni $\mathrm{C}$, Purcell $\mathrm{AE}$, Bouton $\mathrm{CM}$, Pevsner J. High throughput analysis of gene expression in the human brain. J Neurosci Res 2000; 59: 1-10.

18. McGrath J, Feron F, Burne THJ, Mackay-Sim A, Eyles D. The neurodevelopmental hypothesis of schizophrenia; a review of recent developments. Ann Med 2003; 35: 86-93.

19. McGrath JJ, Selten JP. Mental health: don't overlook environment and its risk factors. Nature 2008; 454: 824 .

20. van Os J, Kenis G, Rutten BP. The environment and schizophrenia. Nature 2010; 468: 203-212.

21. McGrath J, Saha S, Welham J, El Saadi O, MacCauley C, Chant D. A systematic review of the incidence of schizophrenia: the distribution of rates and the influence of sex, urbanicity, migrant status and methodology. BMC Med 2004; 2: 13.

22. Cantor-Graae E, Selten JP. Schizophrenia and migration: a meta-analysis and review. Am J Psychiatry 2005; 162: 12-24.

23. Fearon P, Kirkbride JB, Morgan C, Dazzan P, Morgan K, Lloyd T et al. Incidence of schizophrenia and other psychoses in ethnic minority groups: results from the MRC AESOP Study. Psychol Med 2006; 36: 1541-1550.

24. Dealberto MJ. Ethnic origin and increased risk for schizophrenia in immigrants to recent and traditional countries of immigration. Acta Psychiatrica Scandinavica 2010; 121 325-339.

25. Selten JP, Cantor-Graae E. Social defeat: risk factor for schizophrenia? Br J Psychiatry 2005; 187: 101-102.

26. Marcelis M, Navarro-Mateu F, Murray R, Selten JP, van Os J. Urbanization and psychosis: a study of 1942-1978 birth cohorts in The Netherlands. Psychol Med 1998; 28: 871-879.

27. Mortensen PB, Pedersen CB, Westergaard T, Wohlfahrt J, Ewald H, Mors $\mathrm{O}$ et al. Familia and non-familial risk factors for schizophrenia: a population-based study. Schizophr Res 1998; 29: 13.

28. McGrath J. Hypothesis: is low prenatal vitamin $D$ a risk-modifying factor for schizophrenia? Schizophr Res 1999; 40: 173-177.

29. Holick MF. Vitamin D deficiency. N Engl J Med 2007; 357: 266-281.

30. Davies G, Welham J, Chant D, Torrey EF, McGrath J. A systematic review and metaanalysis of Northern Hemisphere season of birth studies in schizophrenia. Schizophr Bull 2003; 29: 587-593.

31. McCann JC, Ames BN. Is there convincing biological or behavioral evidence linking vitamin D deficiency to brain dysfunction? FASEB J 2008; 22: 982-1001.

32. Eyles DW, Smith S, Kinobe R, Hewison M, McGrath JJ. Distribution of the vitamin D receptor and 1alpha-hydroxylase in human brain. J Chem Neuroanat 2005; 29: 21-30.

33. Eyles DW, Feron F, Cui X, Kesby JP, Harms LH, Ko P et al. Developmental vitamin deficiency causes abnormal brain development. Psychoneuroendocrinology 2009; 34(Suppl 1): S247-S257.

34. Dobbs D. Schizophrenia: the making of a troubled mind. Nature 2010; 468: 154-156.

35. Murray RM, Morrison PD, Henquet C, Di Forti M. Cannabis, the mind and society: the hash realities. Nat Rev Neurosci 2007; 8: 885-895.
36. McGrath J, Welham J, Scott J, Varghese D, Degenhardt L, Hayatbakhsh MR et al. Association between cannabis use and psychosis-related outcomes using sibling pair analysis in a cohort of young adults. Arch Gen Psychiatry 2010; 67: 440-447.

37. Sullivan PF, Kendler KS, Neale MC. Schizophrenia as a complex trait: evidence from a meta-analysis of twin studies. Arch Gen Psychiatry 2003; 60: 1187-1192.

38. Shi J, Levinson DF, Duan J, Sanders AR, Zheng Y, Pe'er I et al. Common variants on chromosome 6p22.1 are associated with schizophrenia. Nature 2009; 460: 753-757.

39. Purcell SM, Wray NR, Stone JL, Visscher PM, O'Donovan MC, Sullivan PF et al. Common polygenic variation contributes to risk of schizophrenia and bipolar disorder. Nature 2009; 460: 748-752.

40. Stefansson H, Ophoff RA, Steinberg S, Andreassen OA, Cichon S, Rujescu D et al. Common variants conferring risk of schizophrenia. Nature 2009; 460: 744-747.

41. Wray NR, Visscher PM. Narrowing the boundaries of the genetic architecture of schizophrenia. Schizophr Bull 2010; 36: 14-23.

42. Stone JL, O'Donovan MC, Gurling H, Kirov GK, Blackwood DH, Corvin A et al. Rare chromosomal deletions and duplications increase risk of schizophrenia. Nature 2008; 455 237-241.

43. McClellan JM, Susser E, King MC. Schizophrenia: a common disease caused by multiple rare alleles. Br J Psychiatry 2007; 190: 194-199.

44. Tarpey PS, Smith R, Pleasance E, Whibley A, Edkins S, Hardy C et al. A systematic, largescale resequencing screen of $\mathrm{X}$-chromosome coding exons in mental retardation. Nat Genet 2009; 41: 535-543.

45. Hunt DM, Burnet B. Gene-environment interactions of the eyeless mutant in Drosophila melanogaster. Genet Res 1969; 13: 251-265.

46. Grossman AW, Churchill JD, McKinney BC, Kodish IM, Otte SL, Greenough WT. Experience effects on brain development: possible contributions to psychopathology. J Child Psychol Psychiatry 2003; 44: 33-63.

47. Woolf CM. Does the genotype for schizophrenia often remain unexpressed because of canalization and stochastic events during development? Psychol Med 1997; 27 659-668.

48. Bredy TW, Sun YE, Kobor MS. How the epigenome contributes to the development of psychiatric disorders. Dev Psychobiol 2010; 52: 331-342.

49. Weaver IC, Diorio J, Seckl JR, Szyf M, Meaney MJ. Early environmental regulation of hippocampal glucocorticoid receptor gene expression: characterization of intracellular mediators and potential genomic target sites. Ann NY Acad Sci 2004; 1024: 182-212.

50. Waterland RA. Is epigenetics an important link between early life events and adult disease? Horm Res 2009; 71(Suppl 1): 13-16.

51. Waterland RA, Travisano M, Tahiliani KG. Diet-induced hypermethylation at agouti viable yellow is not inherited transgenerationally through the female. FASEB J 2007; 21: 3380-3385.

52. Brown AS, McGrath JJ. The prevention of schizophrenia. Schizophr Bull 2011; 37 257-261.

53. Licinio J. Translational psychiatry: leading the transition from the cesspool of devastation to a place where the grass is really greener. Translational Psychiatry 2011; 1: e1.

Translational Psychiatry is an open-access journal published by Nature Publishing Group. This work is licensed under the Creative Commons Attribution-Noncommercial-No Derivative Works 3.0 Unported License. To view a copy of this license, visit http://creativecommons.org/licenses/by-nc-nd/3.0/ 